\title{
Considering Mycological Rarities
}

\author{
Byron Breedlove
}

$\mathrm{N}$

either plant nor animal, fungal organisms-including lichen, mildew, mushrooms, molds, rusts, smuts, and yeasts-are found in nearly every possible terrestrial habitat, even aboard the International Space Station. There are millions of species of fungi, and according to the Centers for Disease Control and Prevention, a few hundred fungal species cause illness in people, ranging from allergies and asthma, to skin rashes and infections, to deadly infections of the bloodstream or lungs.

In a 2013 EID article, Mary Brandt and Benjamin Park note the growing number of human infections from traditional and new fungal agents. Factors driving this emergence, they explain, include medical treatments that make immunocompromised patients more susceptible. They also state that "Risk factors such as changes in land use, seasonal migration, international travel, extreme weather, and natural disasters, and the use of azole antifungal agents in largescale agriculture are believed to underlie many of the increases in community-acquired fungal infections."

The recent emergence of Candida auris infections, for instance, underscores those concerns on a broad scale because $C$. auris is often multidrug-resistant, difficult to identify, and causes outbreaks in healthcare settings. A recent study from Finland that reported life-threatening fungal bloodstream infections associated with consuming probiotic supplements that contain Saccharomyces boulardii reveals a route of infection that may represent another mycological issue.

Fungi also have beneficial medicinal and culinary attributes. They were used in traditional medicine long before Alexander Fleming identified and extracted the therapeutic ingredient penicillin from Penicillium in 1928. They have subsequently been used to develop antibiotics, fungicides, anticancer drugs, and cholesterol inhibitors. Mushrooms and truffles are highly desirable foods; yeast is essential for baking, brewing, and fermenting; and molds flavor and color cheeses.

Another attribute of fungi, spalted wood-that is, wood colonized and stained by certain species of

Author affiliation: Centers for Disease Control and Prevention, Atlanta, Georgia, USA

DOI: https://doi.org/10.23201/eid2709.AC2709

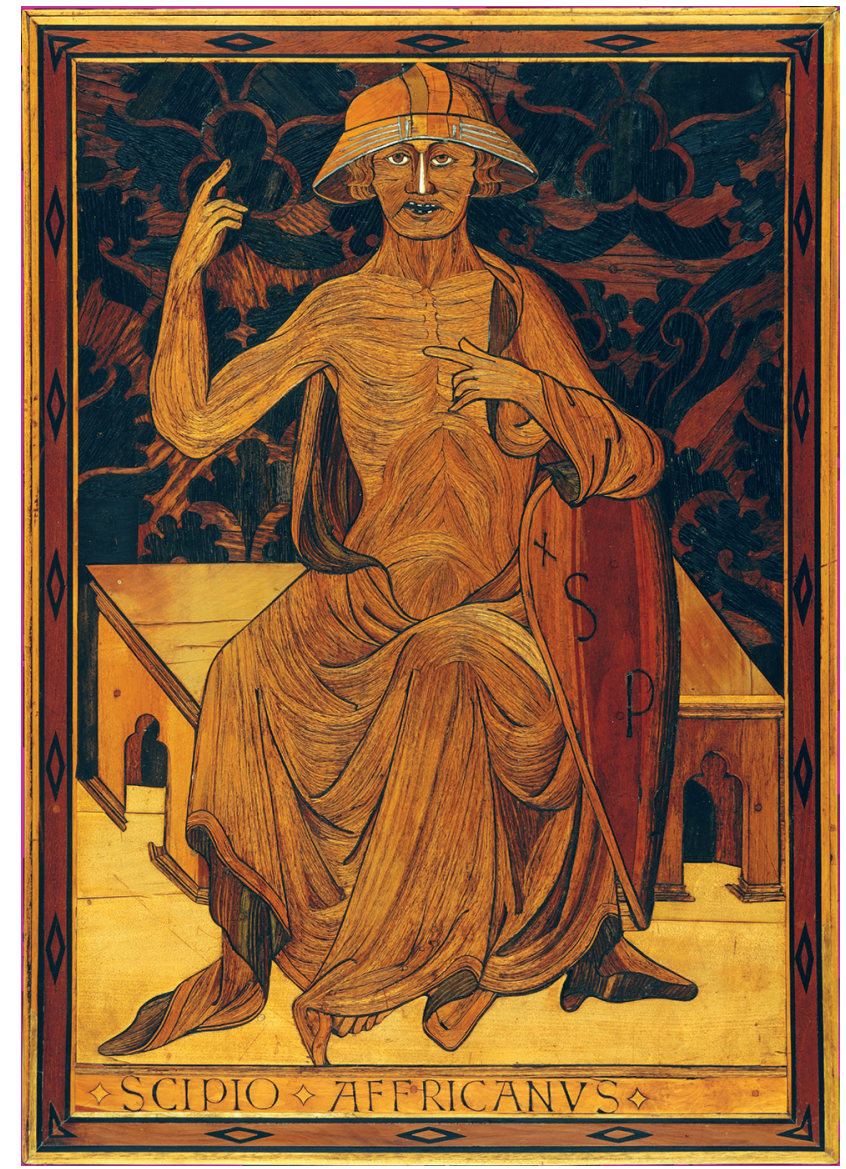

Mattia di Nanni di Stefano (1403-1433), Scipio Africanus ca. 1425-1430. Poplar, bog oak and other wood inlay, rosewood, tin, bone, traces of green coloring, 24.19 in $\times 17.13 \mathrm{in} / 61.5 \mathrm{~cm} \times 43.3$ $\mathrm{cm}$. Public domain image courtesy of The Metropolitan Museum of Art, New York, NY, USA.

fungi-was a prized commodity among European artisans who practiced the form of wood inlaying called intarsia. Spalted wood may be naturally created or stained by an artist; colors may be green, red, yellow, brown, or black. Writer David Elkind explains that green wood discolored by the green elf cup fungus Chlorociboria aeruginascens "happened to fill a lucrative niche in a burgeoning luxury trade, and that made it, for a time at least, as precious as some rare metals."

Intarsia, described as painting with wood to create mosaics as opposed to painting directly onto wood, is 
thought to have originated before the seventh century CE. Its zenith was in Italy during the Renaissance (c. 1400-1600). The Tuscan city of Siena, Italy, known for producing many accomplished painters, was home to several intarsiatori, including Domenico di Niccolo and his apprentice Mattia di Nanni. Intarsiatori inlayed varied shapes, sizes, and species of wood-each with distinct patterns and tones-to fashion decorative items, panels, and elaborate pieces of furniture.

Featured on this month's cover is a wooden panel depicting Roman general Scipio Africanus, crafted by Mattia. According to the Metropolitan Museum of Art, this panel came from what must have been a quite large intarsia bench created for the council chamber of the Palazzo Pubblico in Siena and placed under Simone Martini's fresco the Maestà, a $7.62 \mathrm{~m} \times$ $9.98 \mathrm{~m}$ painting that fills the north wall of the chamber. The bench comprised several panels depicting figures from Roman Republican history considered to be "models of civic virtue, such as the illustrious general Publius Cornelius Scipio Africanus." Scipio is remembered for the strategic and diplomatic skills that enabled him to defeat Hannibal in the Battle of Zama and end the Second Punic War in 202 все.

Mattia portrays Scipio gesturing with his handsperhaps making the point that a leader must follow his head and his heart-and fixing an unyielding gaze on the viewer. The whorls and details in the interlocked wood pieces show muscles, eyes, hair, a draped tunic. A rich, patterned background adds contrast and texture. Noted woodworker Silas Kopf writes that Mattia's skills surpassed those of Domenico, from whom he had learned "how to create a strong graphic presentation through contrast, developing the craft further by laminating small pieces of wood into larger shapes."

Intarsiatori mapped out patterns and colors on paper and then created a matrix or framework to be filled in with different types, shapes, and sizes of wood. Their toolbox included saws, planes, chisels, clamps, knives, pigments, and varnishes. Intarsia projects required large amounts of different colored and textured types of wood, including oak, cypress, walnut, fruitwoods, boxwood, and spindle-wood. The artist would attach sections and pieces of wood called tesserae to the frame, following the paper template, incorporating larger pieces, and filling in with smaller ones to add details and depth. Mattia was among those who used additional materials: his Scipio Africanus features teeth made from bone and a helmet inlaid with metal strips.

Art historian Antoine Wilmering notes that Mattia meticulously tapered the ends of the tesserae, "enabling precise and smooth interweaving of the different, naturally coloured woods. This technique allowed Mattia to create images with carefully modelled details, and some of the inlaid slivers are as fine as a painter's brush." The greenish tints in this panel may be slivers of naturally spalted wood, likely griinfaule or "green oak." As intarsia expanded across Europe, such wood became highly prized. Elkind notes that green wood discolored by the green elf cup fungus C. aeruginascens was "a mycological rarity."

The craft of intarsia continued to evolve, but spalted wood fell into disfavor once inorganic dyes and stains were readily available. Interest in incorporating spalted wood into intarsia was rekindled in the 1950s, and Professor Sara C. Robinson oversees a laboratory at Oregon State University focused on finding new uses for spalted wood not limited to the creative arts. A recent article by Hyde et. al. in the journal Fungal Diversity takes a broader view and examines 50 ways to exploit fungi as an untapped resource, including applications as antibacterials, antimycotics, fungicides, and biofilm inhibitors. Ubiquitous and unique, fungi have a fascinating array of yet unexplored uses.

\section{Bibliography}

1. Brandt ME, Park BJ. Think fungus-prevention and control of fungal infections. Emerg Infect Dis. 2013;19:1688-9. https://doi.org/10.3201/eid1910.131092

2. Centers for Disease Control and Prevention. Fungal diseases [cited 2021 Jul 17]. https://www.cdc.gov/fungal/index.html

3. Elkind D. Exquisite rot: spalted wood and the lost art of intarsia [cited 2021 Jul 8]. https:/ / publicdomainreview.org/ essay/exquisite-rot-spalted-wood-and-the-lost-art-of-intarsia

4. Hyde KD, Xu J, Rapior S, Jeewon R, Lumyong S, Niego AG, et al. The amazing potential of fungi: 50 ways we can exploit fungi industrially. Fungal Divers. 2019;97:1-136. https://doi.org/10.1007/s13225-019-00430-9

5. Jackson FH. Intarsia and marquetry [cited 2021 Jul 15]. https://www.gutenberg.org/files/30215/30215-h/30215-h.htm

6. Kopf S. A marquetry odyssey: historical objects and personal work. Manchester (Vermont): Hudson Hills Press; 2008. p. 31-36, 48 .

7. Metropolitan Museum of Art. Scipio Africanus [cited 2021 Jul 8]. https://www.metmuseum.org/art/collection/ search/208569

8. Rannikko J, Holmberg V, Karppelin M, Arvola P, Huttunen R, Mattila E, et al. Fungemia and other fungal infections associated with use of Saccharomyces boulardii probiotic supplements. Emerg Infect Dis. 2021;27:2043-51.

9. Robinson SC. The fine art of decay [cited 2021 Jul 17]. https://www.americanscientist.org/article/the-fine-artof-decay

10. Wilmering A. Domenico Di Niccolò, Mattia Di Nanni and the development of Sienese intarsia techniques [cited 2021 Jul 21]. https://www.jstor.org/stable/887576

Address for correspondence: Byron Breedlove, EID Journal, Centers for Disease Control and Prevention, 1600 Clifton Rd NE, Mailstop H16-2, Atlanta, GA 30329-4027, USA; email: wbb1@cdc.gov 